\title{
Intra-articular saline injection is as effective as corticosteroids, platelet-rich plasma and hyaluronic acid for hip osteoarthritis pain: a systematic review and network meta-analysis of randomised controlled trials
}

\author{
Aaron Gazendam 다, ${ }^{1}$ Seper Ekhtiari, ${ }^{1}$ Anthony Bozzo, ${ }^{1}$ Mark Phillips, ${ }^{2}$ \\ Mohit Bhandari ${ }^{1}$
}

- Additional material is published online only. To view please visit the journal online (http://dx.doi.org/10.1136/ bjsports-2020-102179).

${ }^{1}$ Orthopedic Surgery, McMaster University, Hamilton, Ontario, Canada

${ }^{2}$ Health Research Methods Evidence and Impact, McMaster University, Hamilton, Ontario, Canada

Correspondence to Dr Aaron Gazendam, Orthopedic Surgery, McMaster University, Hamilton, ON L8S 4L8, Canada; aaron.gazendam@gmail.com

Accepted 23 July 2020 Published Online First 22 August 2020

Check for updates

(C) Author(s) (or their employer(s)) 2021. No commercial re-use. See rights and permissions. Published by BMJ.

To cite: Gazendam A,

Ekhtiari S, Bozzo A

et al. Br J Sports Med

2021:55:256-261.

\section{ABSTRACT}

Objective Intra-articular (IA) injections represent a commonly used modality in the treatment of hip osteoarthritis (OA). Commonly used injections include corticosteroids (CCS), hyaluronic acid (HA) and plateletrich plasma (PRP). A network meta-analysis allows for comparison among more than two treatment arms and uses both direct and indirect comparisons between interventions. The objective of this network meta-analysis is to compare the efficacy of the various IA injectable treatments in treating hip $\mathrm{OA}$ at up to 6 months of follow-up.

Design This is a systematic review and network metaanalysis. Bayesian random-effects model was performed to assess the direct and indirect comparisons of all treatment options.

Data sources PubMed, Embase, Cochrane Central Register of Controlled Trials, Scopus and Web of Science, from inception to October 2019.

Eligibility criteria for selected studies Randomised controlled trials assessing the efficacy of CCS, HA, PRP and placebo in the form of IA saline injection for patients with hip OA.

Results Eleven randomised controlled trials comprising 1353 patients were included. For pain outcomes at both 2-4 and 6 months, no intervention significantly outperformed placebo IA injection. For functional outcomes at both 2-4 and 6 months, no intervention significantly outperformed placebo IA injection.

Regarding change from baseline at 2-4 months and 6 months, pooled data demonstrated that all interventions (including placebo), with the exception of $\mathrm{HA}+\mathrm{PRP}$, led to a clinically important improvement in both pain, exceeding the minimal clinically important difference.

Conclusion Evidence suggests that IA hip saline injections performed as well as all other injectable options in the management of hip pain and functional outcomes.

\section{INTRODUCTION}

Osteoarthritis (OA) is a leading cause of disability worldwide, and its prevalence is on the rise, ${ }^{1}$ a fact exacerbated by an ageing global population. ${ }^{2}$ Total joint arthroplasty (TJA), the definitive treatment for OA of many joints, has been particularly successful and prominent in the treatment of hip and knee OA.
Although largely successful, ${ }^{34}$ TJA represents major surgery that is associated with small but important risk of complications and unsatisfactory results. ${ }^{5}$ Thus, it is important that all non-operative options are explored and exhausted for a patient with hip or knee OA, particularly in younger patients where implant longevity is a real concern. ${ }^{6}$

Non-operative treatment for hip and knee OA consists of a stepwise approach, which in reality is generally deployed in a parallel and multimodal manner. This approach includes weight loss, activity modification, physiotherapy, bracing, oral and topical analgesics including non-steroidal anti-inflammatories, and intra-articular (IA) injections. ${ }^{78}$ A number of less invasive surgical options (eg, arthroscopy, unicompartmental knee arthroplasty and so on) also exist, although their indications in patients with $\mathrm{OA}$ are quite narrow. ${ }^{9} 10$

IA injections, representing the most invasive of the non-surgical treatment options, are often reserved for patients who have exhausted most or all of the other non-operative treatment modalities and who have mild to moderate OA. A wide range of IA injection options exist, including local anaesthetics, corticosteroids (CCS) ${ }^{11}$ hyaluronic acid $(\mathrm{HA}){ }^{11}$ platelet-rich plasma (PRP) ${ }^{12}$ and mesenchymal stem cells. ${ }^{13}$ Although some theoretical and in vitro regenerative potential has been demonstrated with some of the above treatments, ${ }^{14}$ at present time all are aimed at symptom relief rather than disease reversal.

A recent network meta-analysis (NMA) evaluated the efficacy of the various non-operative treatment modalities in knee OA. ${ }^{15}$ Interestingly, they found that all active treatments outperformed placebo, and that HA was most likely to be effective in relieving symptoms and improving function compared with other treatments. Whether these findings are generalisable to injections for hip OA remains largely unclear. Multiple randomised controlled trials (RCTs) have been performed comparing various IA injections in treating hip $\mathrm{OA},{ }^{16}$ providing an opportunity to evaluate whether the efficacy of injections in the knee also leads to clinically important improvements in the hip.

We conducted a systematic review and NMA to examine the efficacy of the various IA injectable treatments in treating hip OA at up to 6 months 
of follow-up. Specifically, our aim was to answer the following questions: (1) Which, if any, IA injections are most likely to be superior to others with regard to pain and function improvements? (2) Which, if any, IA injections have the ability to produce improvements that reach the minimal clinically important difference (MCID) for pain and function? (3) Does industry involvement in trials have any impact on the likelihood of positive trial results?

\section{METHODS}

\section{Protocol and registration}

This review followed the Preferred Reporting Items for Systematic Reviews and Meta-Analyses (PRISMA) and Cochrane Collaboration guidelines for performing and reporting systematic reviews and NMA ((online supplemental appendix 1). ${ }^{17} 18$ This meta-analysis was prospectively registered in the PROSPERO (International Prospective Register of Systematic Reviews) registry.

\section{Eligibility criteria}

The exclusion and inclusion criteria were defined a priori. All randomised and 'quasi-randomised' controlled trials involving skeletally mature human participants with a diagnosis of primary hip OA and which compared at least two different IA injections were included if they reported at least one pain or functional outcome at up to 6 months of follow-up. A diagnosis of OA radiographically using a validated score was required for inclusion. Non-randomised studies, trials that included other joints and did not stratify results by joint, as well as studies reporting on periarticular or intramuscular injections were excluded. When overlapping reports of the same trial were encountered, the most recent trial that was adequately powered for the primary outcome was included.

\section{Search strategy}

A systematic literature search of PubMed, Embase, Cochrane Central Register of Controlled Trials, Scopus and Web of Science from inception to 25 October 2019 examining IA injections for hip OA was performed (online supplemental appendix 2). All searches were limited to RCTs in humans. No language limits were placed on the search. Additionally, available reviews related to the IA injection treatment of hip OA were scanned manually.

\section{Study selection}

Two blinded reviewers (SE and AMG) independently screened all articles at the title/abstract and full-text stages using the Rayyan QCRI software (Qatar Computing Research Institute, Doha, Qatar). ${ }^{19}$ Any discrepancies at the title/abstract stage were resolved by automatic inclusion in the full-text review. All discrepancies at the full-text stage were resolved through consensus. Interobserver agreement for the assessments of study eligibility was calculated using Cohen's kappa ( $\kappa$ ) statistics and the $\kappa$ values were interpreted according to $\mathrm{McHugh} .{ }^{20}$

\section{Data extraction}

A collaborative data extraction form was created (Google, California USA) a priori and piloted prior to formal data extraction. ${ }^{21}$ Extracted data included study characteristics, funding sources of the study, location(s) where patients were recruited, allocation concealment, random sequence generation, blinding, details of intervention(s) and comparator(s) including manufacturer and any pertinent molecular properties (eg, molecular weight) reported, and pain and functional outcome scores.
The outcome measures of interest were pain and function scores reported at baseline, 3 months and 6 months. When 3 -month data were not available, we used the closest data point from 2 to 4 months of follow-up. When an article provided data on more than one pain scale, we referred to a previously described hierarchy of pain-related outcomes for a similar population and extracted the outcome that was highest on this list. ${ }^{22}$ Adverse events were recorded by type and overall incidence for each study and classified as serious or non-serious if reported. Given the significant heterogeneity in reporting of adverse events, no comparative analysis was performed. Corresponding authors of studies with missing data were contacted through ResearchGate and via the email address provided with a request for the data.

\section{Network geometry}

A network plot was created to illustrate the network geometry, including the number of unique interventions, how often they are evaluated and the comparisons made between different treatment options. The network plot contained treatment-specific nodes that were weighted based on the number of studies that investigated that treatment. Weighted connections visually represented the number of studies that compared the two connected nodes.

\section{Study appraisal}

Two reviewers (SE and AMG) independently assessed the risk of bias using the Cochrane Risk of Bias V.2.0 tool. ${ }^{2324}$ Each domain was determined to be at low, high or unclear risk of bias. Each study was then given an overall grade of high risk, moderate risk or low risk of bias based on the Cochrane guidance documents for Risk of Bias V.2.0.

\section{Measures of treatment effect}

Different studies used different patient-reported outcome measures. Thus, as per GRADE (Grading of Recommendation Assessment, Development, and Evaluation) guidelines, all scores for each measured outcome were converted to the scale for the most commonly reported instrument. ${ }^{25}$ The assumption that all outcome measures can be linearly transformed was made. Following conversion of all data to a common scale, a mean difference with $95 \%$ credible intervals was calculated and reported. This allowed for two advantages: an intuitive and easily interpretable mean difference, and the consideration of the concept of MCID. The Visual Analogue Scale (VAS, 0-10) for pain and the Western Ontario and McMaster Universities Osteoarthritis Index (WOMAC) for function (0-68) scale were the most commonly used scales respectively, and all other scales were converted to create a common scale for both pain and function. ${ }^{26}{ }^{27}$ The MCID for pain was set at 1.3 on VAS and 7.1 on the WOMAC function index based on previously defined levels. ${ }^{28}$

Given that most studies did not report change from baseline data, there was inadequate information to allow for calculation or imputation of SD; thus, a direct comparison of change from baseline across interventions could not be performed. For a descriptive, qualitative comparison, weighted mean scores were calculated across studies for each intervention and comparator for pain and function. This was performed using the Cochrane Handbook methods and weighted by sample size; weighted mean scores were calculated at baseline and at each follow-up timepoint. ${ }^{29}$ 


\section{Network meta-analysis}

To assess the direct and indirect evidence of all the IA injection options for hip OA treatment strategies, an NMA was conducted to compare all treatments for two outcomes: pain and function scores, each at 2-4 and 6 months. Regarding HA, we grouped high and low molecular weight formulations together, given that no established differences in outcomes have been found in this population.

Results are presented as mean effect sizes for each outcome. Ranking diagrams and forest plots for each outcome were generated. Surface under the cumulative ranking curve (SUCRA) values were reported. The SUCRA score represents the likelihood that a given treatment will rank first for a specific outcome. We report SUCRA as percentages, where a score closer to $100 \%$ represents a greater chance of that treatment being the best among all treatments studied for that outcome. ${ }^{30}$

\section{Assessment of heterogeneity, inconsistency and transitivity}

To account for the potential heterogeneity of the measured outcomes, a Bayesian framework and a random-effects model were used. Non-informative trials were used. Model convergence was tested using the Gelman statistics, which demonstrated convergence of both models. Transitivity was assessed across each of the treatment comparisons through evaluation of the study characteristics and patient demographics. Global inconsistencies across the network were presented using the $\mathrm{I}^{2}$ value. ${ }^{31-33}$ All statistical analyses were performed using RevMan V.5.3 (Cochrane, London, UK) and R V.3.4.2. ${ }^{23}$ The statistical packages used for the NMA were 'gemtc' and 'rjags' built under R V.3.5.3.

\section{Dealing with missing data}

If the mean or SD was missing, the mean score at each timepoint was calculated by subtracting the mean difference from the baseline score, and the SD was imputed using the SD of the other included studies as per the Cochrane Handbook. ${ }^{29}$

\section{Additional analyses}

A meta-regression was performed to assess the effect of direct industry sponsorship on the effect size reported in a trial. A sensitivity analysis was also performed by assessing HA by molecular weight, opposed to as a class. High molecular weight HA (HMWHA) was considered over $3000 \mathrm{kDa}$ and low molecular weight HA (LMWHA) was any formulation below that weight.

\section{RESULTS}

\section{Study selection}

The search strategy retrieved 1782 related articles after duplicates were removed. After title/abstract review, the full texts of 61 potentially eligible articles were reviewed (figure 1). Of the 61 full-text articles reviewed, 11 studies met the inclusion criteria for NMA. ${ }^{34-44}$ Study characteristics can be found in table 1 . The agreement between the two blinded reviewers was moderate ( $\kappa=0.6,95 \% \mathrm{CI} 0.47$ to 0.72 ) at the title/abstract stage and almost perfect ( $\kappa=0.96,95 \%$ CI 0.88 to 1.00$)$ at the fulltext stage. At the full-text stage, there was disagreement about the inclusion of only one study, which was resolved through consensus.

\section{Characteristics of included studies}

All 11 trials included retrievable data for pain and function scores. Nine studies had data available for 2-4 months of follow-up, while six studies had data available for comparison at 6 months of

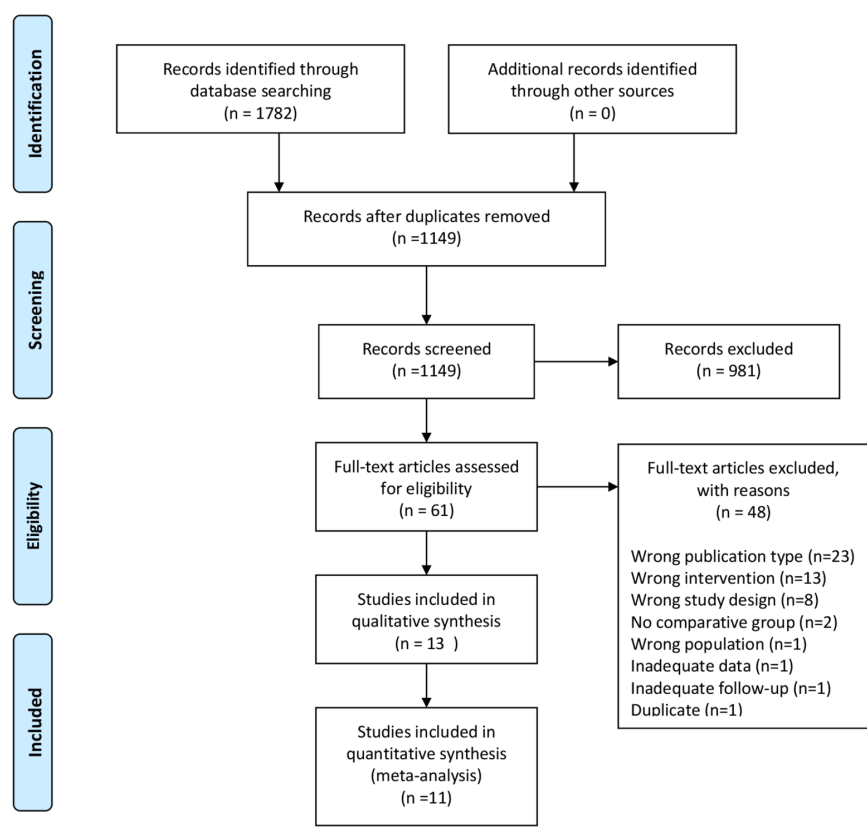

Figure 1 Flow diagram of study selection.

follow-up. The median trial sample was 80 (range, 42-305). One study had four comparative groups, two studies had three comparative groups and the remaining eight studies had two comparative groups each. Figure 2 demonstrates the network of all treatment comparisons for both pain and functional outcomes. Included trials involved a total of 1353 patients with a mean age of $64 \pm 9.5$ years; the proportion of women was 54\% (range, 19\%-66\%). Treatment groups included IA placebo injection with or without local anaesthetic $(n=314)$, HA $(n=596)$, CCS $(n=237)$, PRP $(n=155)$, a combined HA and PRP injection (HA+PRP, $\mathrm{n}=31$ ), and a control group with no injection $(\mathrm{n}=20)$.

The risk of bias summary for each included trial is available in online supplementary figure 1 . Five studies were found to be of low risk of bias, three studies of moderate risk and three studies of high risk of bias. Overall, there was highest risk of bias due to deviations from the intended interventions and missing outcome data.

\section{Pain}

None of the hip injections demonstrated significant improvement in VAS pain scores when compared with saline hip injection at 2-4 months ( 9 trials, 968 patients) or 6 months ( 9 trials, 995 patients) (figure 3, online supplementary figure 2). Regarding change from baseline at $2-4$ months and 6 months, pooled data demonstrated that all interventions (including placebo), with the exception of HA+PRP and the control group, led to a clinically important improvement in pain, exceeding the MCID of 1.3 (figure 3).

\section{Function}

None of the hip injections demonstrated significant improvement in function scores when compared with saline hip injection at 2-4 months (9 trials, 968 patients) or 6 months (9 trials, 995 patients) (figure 4, online supplementary figure 3). Regarding change from baseline at $2-4$ months and 6 months, pooled data demonstrated that all interventions (including placebo), with the exception of HA+PRP and the control group, led to a clinically important improvement in functional scores, exceeding the MCID of 7.1 (figure 4). 
Table 1 Study and patient characteristics

\begin{tabular}{|c|c|c|c|c|c|c|c|c|c|c|}
\hline Study (year) & $\begin{array}{l}\text { Study } \\
\text { design }\end{array}$ & $\begin{array}{l}\text { Level of } \\
\text { evidence }\end{array}$ & $\begin{array}{l}\text { Interventions } \\
\text { (n, patients) }\end{array}$ & $\begin{array}{l}\text { Total } \\
\text { patients }\end{array}$ & Female (\%) & $\begin{array}{l}\text { Mean age } \\
( \pm S D)\end{array}$ & $\begin{array}{l}\text { Follow-up } \\
\text { (weeks) }\end{array}$ & $\mathrm{OA}$ grade & $\begin{array}{l}\text { VAS pain scores ( } 2-4 \\
\text { months, } 6 \text { months) }\end{array}$ & $\begin{array}{l}\text { WOMAC function } \\
\text { scores ( } 2-4 \text { months, } \\
6 \text { months) }\end{array}$ \\
\hline Atchia et al $(2011)^{34}$ & RCT & 1 & $\begin{array}{l}\text { HA (19) } \\
\text { NS (19) } \\
\text { CCS (19) } \\
\text { CTL (20) }\end{array}$ & 77 & $43(56)$ & $68.5(8.3)$ & 8 & $\begin{array}{l}\text { Croft } \\
\text { I-IV }\end{array}$ & $\begin{array}{l}\text { HA }(4.65, N R) \\
\text { NS }(5.8, N R) \\
\text { CCS }(4.3, N R) \\
\text { CTL }(5.5, N R)\end{array}$ & $\begin{array}{l}(35.4, N R) \\
(41.3, N R) \\
(31.8, N R) \\
(40.1, N R)\end{array}$ \\
\hline Battaglia et al $(2013)^{35}$ & RCT & 1 & $\begin{array}{l}\text { PRP (50) } \\
\text { HA (50) }\end{array}$ & 100 & $37(37)$ & $53.5(12)$ & $12-24$ & $\begin{array}{l}\text { K-L } \\
\text { II-IV }\end{array}$ & $\begin{array}{l}\operatorname{PRP}(3.8,4.3) \\
\text { HA }(3.8,4.0)\end{array}$ & $\begin{array}{l}(18.5,20.3) \\
(15.6,16.5)\end{array}$ \\
\hline Brander et al (20198) $)^{37}$ & RCT & 1 & $\begin{array}{l}\text { HA (182) } \\
\text { NS (175) }\end{array}$ & 357 & $210(59)$ & $60.3(9.4)$ & $12-24$ & $\begin{array}{l}\mathrm{K}-\mathrm{L} \\
\mathrm{II}-\mathrm{III}\end{array}$ & $\begin{array}{l}\text { HA }(4.0,4.1) \\
\text { NS }(3.8,4)\end{array}$ & $\begin{array}{l}(29.9,28.8) \\
(28.3,29.3)\end{array}$ \\
\hline Dallari et al (2016) & RCT & 1 & $\begin{array}{l}\text { PRP (44) } \\
\text { HA (36) } \\
\text { PRP+HA (31) }\end{array}$ & 111 & $53(48)$ & NR & $8-24$ & $\begin{array}{l}K-L \\
I-I V\end{array}$ & $\begin{array}{l}\operatorname{PRP}(2.0,2.0) \\
\operatorname{HA}(3.4,5.3) \\
\operatorname{PRP}+H A(3.4,3.9)\end{array}$ & $\begin{array}{l}(17.5,17.3) \\
(25.5,26.9) \\
(28.7,28.1)\end{array}$ \\
\hline Di Sante et al $(2016)^{39}$ & RCT & I & $\begin{array}{l}\text { PRP (21) } \\
\text { HA (22) }\end{array}$ & 43 & $23(53)$ & $72.5(7)$ & 16 & $\begin{array}{l}\text { K-L } \\
\text { II-III }\end{array}$ & $\begin{array}{l}\text { PRP }(5.4, N R) \\
\text { HA }(2.0, N R)\end{array}$ & $\begin{array}{l}(50.8, N R) \\
(28.4, N R)\end{array}$ \\
\hline Doria et al $(2017)^{40}$ & RCT & I & $\begin{array}{l}\text { PRP (40) } \\
\text { HA (40) }\end{array}$ & 80 & NR & $67.7(5.2)$ & 24 & $\begin{array}{l}K-L \\
I-I I\end{array}$ & $\begin{array}{l}\text { PRP }(N R, 6.3) \\
\text { HA (NR, 6.3) }\end{array}$ & $\begin{array}{l}(\mathrm{NR}, 12.3) \\
(\mathrm{NR}, 11.3)\end{array}$ \\
\hline Lambert et al $(2007)^{41}$ & RCT & 1 & $\begin{array}{l}\operatorname{CCS}(31) \\
\text { NS (21) }\end{array}$ & 52 & $31(60)$ & $61.3(11)$ & 8 & $\begin{array}{l}K-L \\
\text { I-IV }\end{array}$ & $\begin{array}{l}\operatorname{CCS}(3.1, N R) \\
\text { NS }(6.1, N R)\end{array}$ & $\begin{array}{l}(24.2, \mathrm{NR}) \\
(42.7, \mathrm{NR})\end{array}$ \\
\hline Migliore et al (2009) ${ }^{42}$ & RCT & 1 & $\begin{array}{l}\text { HA (22) } \\
\text { NS (20) }\end{array}$ & 44 & $20(48)$ & $67.5(8.8)$ & $12-24$ & $\begin{array}{l}\text { K-L } \\
\text { II-IV }\end{array}$ & $\begin{array}{l}\text { HA }(4.3,4.5) \\
\text { NS }(4.5,5.0)\end{array}$ & $\begin{array}{l}(35.0,26.8) \\
(44.4,43.6)\end{array}$ \\
\hline Qvistgaard et al (2006) ${ }^{43}$ & RCT & 1 & $\begin{array}{l}\text { HA (33) } \\
\text { NS (36) } \\
\text { CCS (32) }\end{array}$ & 101 & $65(64)$ & $66(11.3)$ & 12 & $\begin{array}{l}K-L \\
I-I V\end{array}$ & $\begin{array}{l}\text { HA }(3.8, N R) \\
\text { NS }(3.7, N R) \\
\text { CCS }(3.5, N R)\end{array}$ & $\begin{array}{l}(23.4, N R) \\
(23.8, N R) \\
(23.6, N R)\end{array}$ \\
\hline Richette et al (2009) ${ }^{44}$ & RCT & I & $\begin{array}{l}\text { HA (42) } \\
\text { NS (43) }\end{array}$ & 85 & $50(59)$ & $59(11.4)$ & 12 & $\begin{array}{l}\text { K-L } \\
\text { II-III }\end{array}$ & $\begin{array}{l}\text { HA }(5.1, N R) \\
\text { NS }(5.1, N R)\end{array}$ & $\begin{array}{l}(30.3, N R) \\
(29.9, N R)\end{array}$ \\
\hline Spitzer et al $(2010)^{36}$ & RCT & 1 & $\begin{array}{l}\text { HA (150) } \\
\text { CCS (155) }\end{array}$ & 305 & $154(51)$ & $59(11.5)$ & 24 & $\begin{array}{l}\text { K-L } \\
\text { II-III }\end{array}$ & $\begin{array}{l}\text { HA (NR, 4.4) } \\
\text { CCS (NR, 4.8) }\end{array}$ & $\begin{array}{l}(N R, 34.4) \\
(N R, 40.0)\end{array}$ \\
\hline
\end{tabular}

CCS, corticosteroids; CTL, control (no injection); HA, hyaluronic acid; I, level I evidence; K-L, Kellgren-Lawrence; NR, not recorded; NS, normal saline; OA, osteoarthritis; PRP, platelet-rich plasma; RCT, randomised controlled trial; VAS, Visual Analogue Scale; WOMAC, Western Ontario and McMaster Osteoarthritis Index.

\section{SUCRA rankings and pairwise comparisons}

For pain reduction at $2-4$ months, CCS had the largest probability of being the best treatment option in our NMA (SUCRA=90\%) (figure 5). At 6 months, PRP had the largest probability of being the best treatment option in our network (SUCRA=71.5\%). The overall ranking based on SUCRA is shown in table 2. League tables demonstrating the pairwise comparison of all treatment options for pain scores are available in online supplementary figure 4 .

Regarding improvements in functional scores, CCS had the largest probability of being the best treatment in our network (SUCRA $=90.1 \%$ ) at 2-4 months. At 6 months, HA had the largest probability of being the best treatment in our network (SUCRA $=65.1 \%)$. The overall ranking based on SUCRA is shown in table 2. League tables demonstrating the pairwise comparison of all treatment options for functional scores are available in online supplementary figure 5 . The remaining rank probability plots

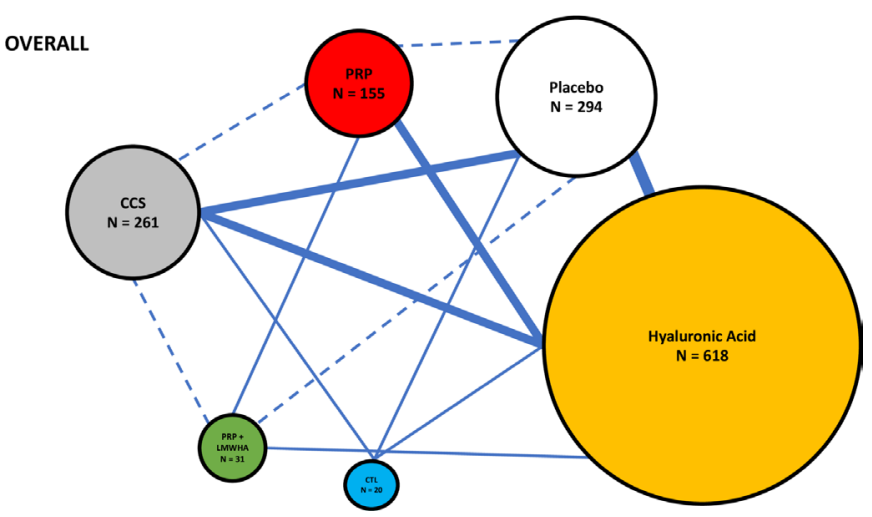

Figure 2 Overall network diagram for all treatment comparisons for both pain and functional outcomes. CCS, corticosteroids; CTL, control; LMWHA, low molecular weight hyaluronic acid; PRP, platelet-rich plasma.

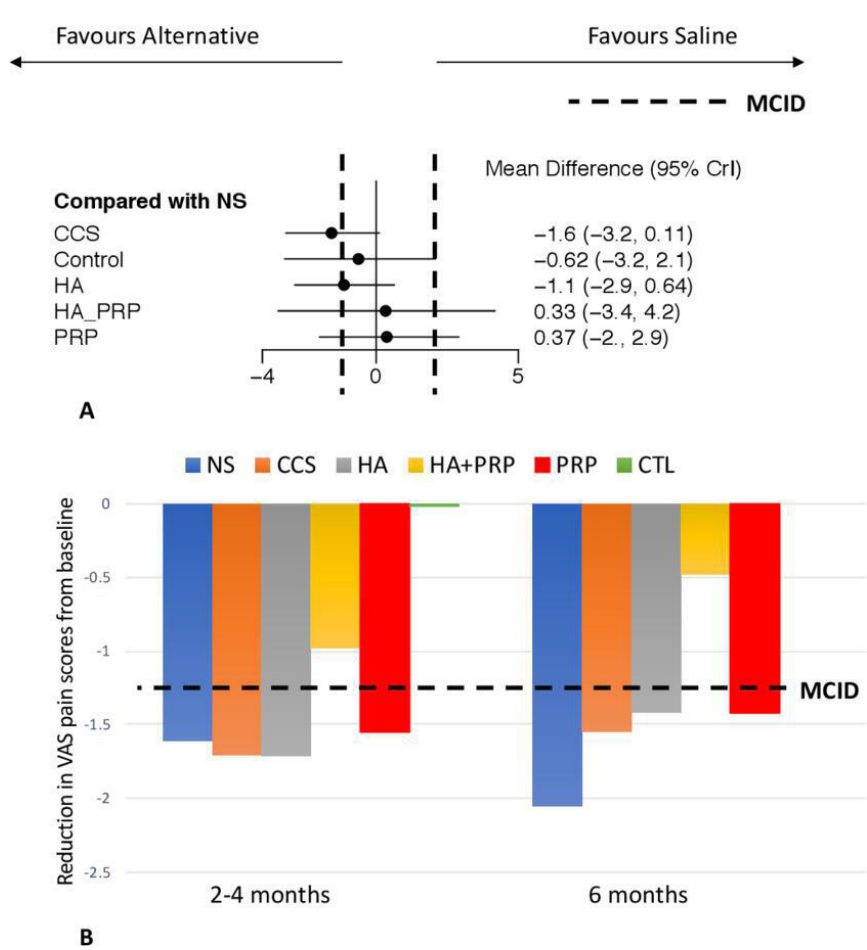

Figure 3 (A) Forest plot diagram showing the mean difference for changes in VAS pain scores compared with placebo at 2-4 months of follow-up. (B) Pooled weighted mean change in pain outcomes and associated MCID of 1.3 for both 2-4 months and 6 months of follow-up. CCS, corticosteroid; Crl, credible interval; CTL, control; HA, hyaluronic acid; MCID, minimal clinically important difference; NS, normal saline; PRP, platelet-rich plasma; VAS, Visual Analogue Scale. 
Favours Saline
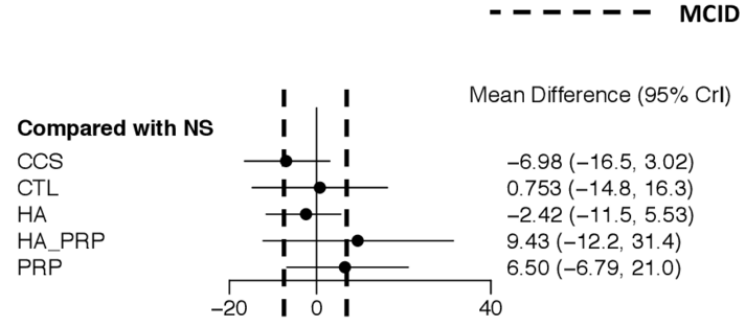

A

NS CCS HA HA+PRP PRP CTL

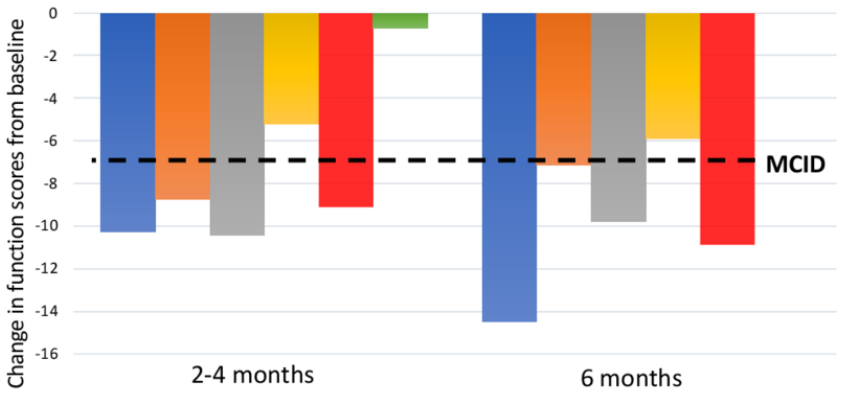

B

Figure 4 (A) Forest plot diagram showing the mean difference for changes in WOMAC function scores compared with placebo at 2-4 months of follow-up. (B) Pooled, weighted mean change in functional outcomes and associated MCID of 7.1 for both 2-4 months and 6 months of follow-up. CCS, corticosteroids; Crl, credible interval; CTL, control; $\mathrm{HA}$, hyaluronic acid; $\mathrm{MCID}$, minimal clinically important difference; NS, normal saline; PRP, platelet-rich plasma; WOMAC, Western Ontario and McMaster Universities Osteoarthritis Index.

(rankograms) for pain scores at 6 months and functional outcomes are available in online supplementary figure 6.

\section{Sensitivity analysis}

The HA formulations were divided into high and low molecular weight with a cut-off of $3000 \mathrm{kDa}$, and an NMA was run for both

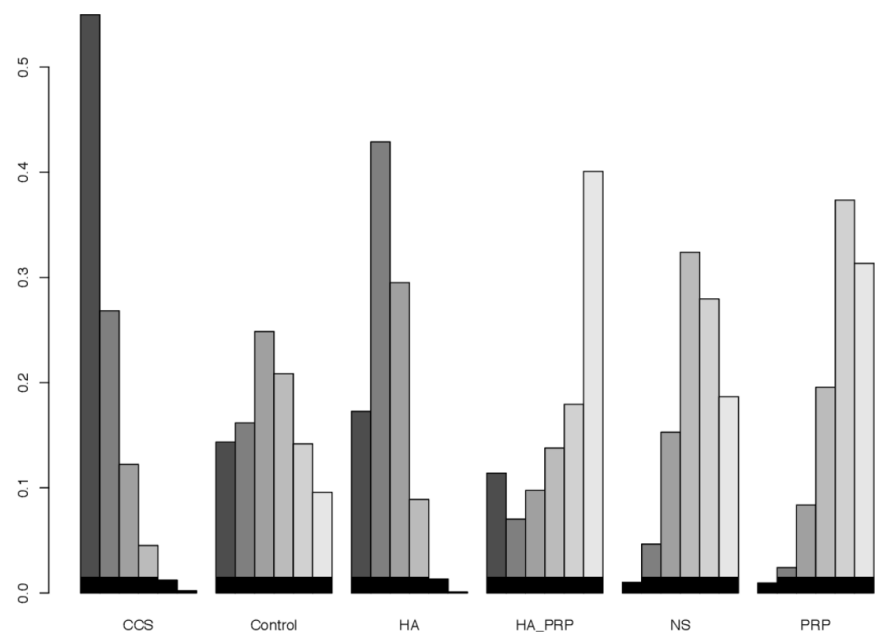

Figure 5 Rankogram illustrating the empirical probabilities that each treatment is ranked first through sixth (left to right) for VAS pain scores at 2-4 months of follow-up. CCS, corticosteroids; HA, hyaluronic acid; NS, normal saline; PRP, platelet-rich plasma; VAS, Visual Analogue Scale. pain and functional outcomes at both timepoints. The analyses demonstrated that CCS still had the largest probability at 2-4 months of follow-up but was not statistically better than placebo.

\section{Meta-regression}

A total of six trials $(n=967)$ received funding from an industry manufacturer of one of the interventions being studied. Metaregression was performed to determine if the observed treatment effects were similar between the trials which received industry funding and those that did not. No significant effect of industry sponsorship was observed for pain reduction at any timepoint.

\section{Safety profile}

Adverse reactions were recorded for 10 out of 11 studies (1273). A total of $143(11.2 \%)$ adverse events that were potentially or probably related to the treatment were recorded. There were 24 (1.9\%) major adverse events that were possibly or probably related to the treatment, including the following: discontinued due to adverse events $(n=20)$, deep vein thrombosis $(n=1)$, rapid progression of OA $(n=1)$, post hip arthroplasty infection $(n=1)$ and superficial haematoma $(n=1)$.

\section{DISCUSSION}

The key findings of this systematic review and NMA demonstrate that compared with placebo, no IA injections demonstrate a statistically significant difference at up to 6 months postinjection for patients with hip OA. Perhaps most importantly, while changes from baseline did reach MCID for most interventions, this was also true of placebo, which often performed similarly to, if not better than, the active treatments.

Interestingly, the recent NMA on knee OA had found a benefit for IA HA and CCS in treating knee OA, although the MCID was not considered. ${ }^{15}$ Furthermore, a recent review of the literature suggested that HMWHA would outperform LMWHA in knee $\mathrm{OA}^{45}$ In the present study on hip OA, no treatment was found to have a clinically meaningful benefit beyond placebo, and a sensitivity analysis demonstrated no significant difference between HMWHA compared with LMWHA. This represents an interesting contrast, yet the reasons for which are not yet clear. The reason for the superiority of HMWHA in knee OA is unclear, although it has been proposed to be related to greater viscosity and thus the ability to act as a lubricating substance. ${ }^{45}$ Theoretically, it is possible that the ball-and-socket morphology of the hip, compared with the relatively more flat surfaces of the knee joint, makes this distinction less important in the hip, although this is not currently supported by any available evidence. Another contrasting feature with the previous NMA on knee OA is the fact that the prior study included oral medications in their network. In the present study, a choice was made to specifically only include IA treatments. This decision was made to reflect clinical practice: it is almost never the case that a physician is deciding between an oral medication and an IA injection, and is forced to choose only one. Much more likely, the patient has already received oral analgesics in the period leading up to the decision to proceed with an IA injection, and the more clinically pertinent question is which injectable treatment to choose from the many options available.

IA injections are currently a mainstay of non-operative treatment in hip OA, although perhaps not as commonly used as they are in knee OA. ${ }^{46}$ Another contrasting feature from knee OA is the need for image guidance, which can represent an added cost to the patient, the healthcare system or both. In a time of rising healthcare costs globally, ${ }^{47}$ all healthcare interventions are judged not only on efficacy, but also on cost-effectiveness. Thus, interventions that, as 
Table 2 SUCRA values and heterogeneity

\begin{tabular}{llllllll}
\hline SUCRA value & & & & & & \\
\hline Pain & CCS & CTL & HA & NS & PRP & HA+PRP & I $^{2}(\%)$ \\
\hline 2-4 months & 0.858 & 0.534 & 0.731 & 0.325 & 0.232 & 0.320 & 78.5 \\
6 months & 0.320 & NR & 0.497 & 0.536 & 0.715 & 0.431 & 56.6 \\
Function & & & & & & & \\
2-4 months & 0.901 & 0.447 & 0.698 & 0.510 & 0.210 & 0.196 & 52.7 \\
6 months & 0.281 & NR & 0.651 & 0.359 & 0.581 & 0.628 & 67.4 \\
\hline
\end{tabular}

CCS, corticosteroids; CTL, control; HA, hyaluronic acid; NR, not recorded; NS, normal saline; PRP, platelet-rich plasma; SUCRA, surface under the cumulative ranking curve.

of yet, seem unable to produce benefits that exceed the benefits of placebo are unlikely to be cost-effective, although a formal costutility analysis is beyond the scope of this study. Nonetheless, it is difficult to counsel the individual patient in these scenarios. As with arthroscopy for patients with OA, some individual patients certainly do benefit, and the benefit of placebo injection (or sham surgery) in and of itself can provide important improvement for some patients. ${ }^{48}$ When the only other option is TJA, many patients may reasonably choose the less invasive option, even if the best evidence does not promise any benefit. In this case, the principle of beneficence must be balanced with the principle of justice, particularly when the treatment is being covered, fully or in part, through publicly funded insurance.

There are inherent limitations when performing NMA.49 50 One particular area of controversy is in the utilisation of SUCRAbased treatment rank estimates. SUCRA rankings disproportionately rank interventions with larger uncertainty in their effect sizes higher. The number of studies per treatment comparison, the network geometry, the estimated treatment effect size and the exclusions of trials can all impact the rank probabilities. ${ }^{33} 51$ Rankings also suggest differences in the efficacy of various treatments even when no statistically or clinically relevant differences have been found. Given that no intervention significantly improved outcomes when compared to placebo, the SUCRA rankings should be taken with considerable reservations. ${ }^{50}$

\section{What is already known}

- Intra-articular (IA) injections, representing the most invasive of the non-surgical treatment options, are often reserved for patients who have exhausted most or all of the other non-operative treatment modalities and who have mild to moderate osteoarthritis (OA).

- A wide range of IA injection options exist, including local anaesthetics, corticosteroids, hyaluronic acid, platelet-rich plasma and mesenchymal stem cells.

- While multiple randomised controlled trials have been performed comparing various IA injections in treating hip OA it remains unclear which, if any, is most effective.

- A number of randomised controlled trials tout 'statistically significant' patient improvement, and clinically significant results remain much more elusive.

\section{What are the new findings}

- The current review demonstrates that no currently available intra-articular injections provide significant improvement in pain and function when compared with placebo at short-term follow-up.
The advantage of an NMA over a traditional meta-analysis is the ability to make both direct and indirect comparisons between multiple potential treatment options, thereby allowing the comparison of similar but distinct treatments for a given condition that may have never been compared directly in a clinical setting. ${ }^{52}$ In this way, an NMA can help to inform clinical practice and generate hypotheses for the design of RCTs by identifying those treatments most likely to be efficacious, and similarly those most likely to be futile.

A primary strength of this study is the inclusion of only level I evidence, which allows the execution of an NMA and the drawing of conclusions about comparative efficacy. In addition, strict adherence to rigorous methodology and Cochrane and PRISMA guidelines allows the work to be both reliable and reproducible. In addition, prospective protocol registration and rapid completion of the study ensure transparency and relevance.

Limitations of this NMA include the relatively small sample sizes of the included RCTs, which should be considered when designing future trials. As well, no conclusions can be drawn about the comparative efficacy of IA injections and other forms of nonoperative treatment for hip OA. However, as previously discussed, this is rarely a binary choice that is made in real clinical practice.

As is the case with most topics in orthopaedic surgery and indeed in medicine, future large RCTs are needed to assess the efficacy (or lack thereof) of IA injection in the treatment of hip OA. Future RCTs should be designed rigorously with large enough sample sizes to definitively answer these questions, and multiarm trials may represent the most efficient way to achieve this goal.

Contributors All authors have made substantial contributions to all of the following: conception and design of the study, or acquisition of data, or analysis and interpretation of data; drafting the article or revising it critically for important intellectual content; and final approval of the version to be submitted. All authors have approved the submission of this manuscript. The manuscript, including related data, figures and tables, has not been previously published and is not under consideration elsewhere.

Funding The authors have not declared a specific grant for this research from any funding agency in the public, commercial or not-for-profit sectors.

Competing interests MB reports personal fees from AgNovos Healthcare, personal fees and other from Sanofi Aventis, personal fees and other from Smith \& Nephew, personal fees from Stryker, grants from DJ Orthopedics, and other from Ferring Pharmaceuticals, outside the submitted work. No other authors report competing interests.

Patient consent for publication Not required.

Provenance and peer review Not commissioned; externally peer reviewed.

ORCID iD

Aaron Gazendam http://orcid.org/0000-0002-2615-4919

\section{REFERENCES}

1 Hunter DJ, Bierma-Zeinstra S. Osteoarthritis. Lancet 2019.

2 Sander $\mathrm{M}, \mathrm{Oxlund} \mathrm{B}$, Jespersen $\mathrm{A}$, et al. The challenges of human population ageing Age Ageing 2015;44:185-7.

3 Khanduja V. Total hip arthroplasty in 2017-Current concepts and recent advances. Indian J Orthop 2017 
4 Varacallo M, Johanson NA. Total Knee Arthroplasty (TKA) Techniques 2018.

5 Gronbeck C, Cote MP, Lieberman JR, et al. Risk stratification in primary total joint arthroplasty: the current state of knowledge. Arthroplast Today 2019;5:126-31.

6 Schreurs BW, Hannink G. Total joint arthroplasty in younger patients: heading for trouble? Lancet 2017;389:1374-5.

7 Vaishya R, Pariyo GB, Agarwal AK, et al. Non-Operative management of osteoarthritis of the knee joint. J Clin Orthop Trauma 2016;7:170-6.

8 Marks R. Non-Operative management of hip osteoarthritis. Pain Stud Treat 2015.

9 Lustig S, Donell ST, Pagenstert G, et al. Unicompartmental knee arthroplasty. In: ESSKA instructional course lecture book. Glasgow, 2018.

10 Siemieniuk RAC, Harris IA, Agoritsas T, et al. Arthroscopic surgery for degenerative knee arthritis and meniscal tears: a clinical practice guideline. BMJ 2017;357:j1982.

11 Migliore A, Granata M. Intra-Articular use of hyaluronic acid in the treatment of osteoarthritis. Clin Interv Aging 2008;3:365-9.

12 Bennell KL, Hunter DJ, Paterson KL. Platelet-Rich plasma for the management of hip and knee osteoarthritis. Curr Rheumatol Rep 2017;19:24.

13 Dall'Oca C, Breda S, Elena N, et al. Mesenchymal stem cells injection in hip osteoarthritis: preliminary results. Acta Biomed 2019;90:75-80.

14 Kennedy MI, Whitney K, Evans T, et al. Platelet-Rich plasma and cartilage repair. Curr Rev Musculoskelet Med 2018;11:573-82.

15 Bannuru RR, Schmid CH, Kent DM, et al. Comparative effectiveness of pharmacologic interventions for knee osteoarthritis: a systematic review and network meta-analysis. Ann Intern Med 2015;162:46-54.

16 Wu B, Li Y-M, Liu Y-C. Efficacy of intra-articular hyaluronic acid injections in hip osteoarthritis: a meta-analysis of randomized controlled trials. Oncotarget. In Press 2017:8:86865-76

17 Higgins JPT, Altman DG, Gøtzsche PC, et al. The Cochrane collaboration's tool for assessing risk of bias in randomised trials. Br Med J 2011;343:d5928-93.

18 Hutton B, Salanti G, Caldwell DM, et al. The PRISMA extension statement for reporting of systematic reviews incorporating network meta-analyses of health care interventions: checklist and explanations. Ann Intern Med : 2015;162:777.

19 Ouzzani M, Hammady H, Fedorowicz Z, et al. Rayyan-a web and mobile APP for systematic reviews. Syst Rev 2016;5:210.

20 McHugh ML. Interrater reliability: the kappa statistic. Biochem Med 2012;22:276-82

21 Graham MJ, Borgen J. Google sheets. In: Google tools meets middle school 2018

22 Juhl C, Lund H, Roos EM, et al. A hierarchy of patient-reported outcomes for metaanalysis of knee osteoarthritis trials: empirical evidence from a survey of high impact journals. Arthritis 2012;2012:1-17.

23 The Nordic Cochrane Centre. Review manager (RevMan). Cochrane Collab 2014.

24 Sterne JAC, Savović J, Page MJ, et al. Rob 2: a revised tool for assessing risk of bias in randomised trials. BMJ 2019;366:14898.

25 Guyatt GH, Oxman AD, Schünemann HJ, et al. Grade guidelines: a new series of articles in the Journal of clinical epidemiology. J Clin Epidemiol 2011;64:380-2.

26 Boonstra AM, Schiphorst Preuper HR, Reneman MF, et al. Reliability and validity of the visual analogue scale for disability in patients with chronic musculoskeletal pain. Int J Rehabil Res 2008:31:165-9.

27 Roos MK, Lohmander E LS. WOMAC osteoarthritis index: reliability, validity, and responsiveness in patients with arthroscopically assessed osteoarthritis.. Scand J Rheumatol 1999

28 Devji T, lorio A, Guyatt GH. PROMID 2019.

29 Cochrane Handbook for systematic reviews of interventions 2019.

30 Chaimani A, Higgins JPT, Mavridis D, et al. Graphical tools for network meta-analysis in STATA. PLoS One 2013;8:e76654.
31 Higgins JPT, Thompson SG, Deeks JJ, et al. Measuring inconsistency in meta-analyses. BMJ 2003:327:557-60.

32 Higgins J, Green S. Cochrane Handbook for Systematic Reviews of Interventions Version 5.1.0 [updated March 2011]. Cochrane Collab 2011.

33 Veroniki AA, Straus SE, Rücker G, et al. Is providing uncertainty intervals in treatment ranking helpful in a network meta-analysis? J. Clin. Epidemiol 2018.

34 Atchia I, Kane D, Reed MR, et al. Efficacy of a single ultrasound-guided injection for the treatment of hip osteoarthritis. Ann Rheum Dis 2011;70:110-6.

35 Battaglia M, Guaraldi F, Vannini F, et al. Efficacy of ultrasound-guided intra-articular injections of platelet-rich plasma versus hyaluronic acid for hip osteoarthritis. Orthopedics 2013;36:e1501-8.

36 Spitzer Al, Bockow BI, Brander VA, et al. Hylan G-F 20 improves hip osteoarthritis: a prospective, randomized study. Phys Sportsmed 2010;38:35-47.

37 Brander V, Skrepnik N, Petrella RJ, et al. Evaluating the use of intra-articular injections as a treatment for painful hip osteoarthritis: a randomized, double-blind, multicenter, parallel-group study comparing a single 6-mL injection of hylan G-F 20 with saline. Osteoarthr Cartil 2019;27:59-70.

38 Dallari D, Stagni C, Rani N, et al. Ultrasound-Guided injection of platelet-rich plasma and hyaluronic acid, separately and in combination, for hip osteoarthritis: a randomized controlled study. Am J Sports Med 2016;44:664-71.

39 Di Sante L, Villani C, Santilli V, et al. Intra-Articular hyaluronic acid vs platelet-rich plasma in the treatment of hip osteoarthritis. Med Ultrason 2016;18:463.

40 Doria C, Mosele G, Caggiari G, et al. Treatment of early hip osteoarthritis: ultrasoundguided platelet rich plasma versus hyaluronic acid injections in a randomized clinical trial. Joints 2017;05:152-5.

41 Lambert RGW, Hutchings EJ, Grace MGA, et al. Steroid injection for osteoarthritis of the hip: a randomized, double-blind, placebo-controlled trial. Arthritis Rheum 2007:56:2278-87.

42 Migliore A, Massafra U, Bizzi E, et al. Comparative, double-blind, controlled study of intra-articular hyaluronic acid (Hyalubrix) injections versus local anesthetic in osteoarthritis of the hip. Arthritis Res Ther 2009;11:R183.

43 Qvistgaard E, Christensen R, Torp-Pedersen S, et al. Intra-Articular treatment of hip osteoarthritis: a randomized trial of hyaluronic acid, corticosteroid, and isotonic saline. Osteoarthr Cartil 2006;14:163-70.

44 Richette P, Ravaud P, Conrozier T, et al. Effect of hyaluronic acid in symptomatic hip osteoarthritis: a multicenter, randomized, placebo-controlled trial. Arthritis Rheum. In Press 2009;60:824-30.

45 Bhandari M, Bannuru RR, Babins EM, et al. Intra-Articular hyaluronic acid in the treatment of knee osteoarthritis: a Canadian evidence-based perspective. Ther Adv Musculoskelet Dis 2017;9:231-46.

46 Stephens MB, Beutler Al, O'Connor FG. Musculoskeletal injections: a review of the evidence. Am Fam Physician 2008.

47 OECD. Fiscal sustainability of health systems: bridging health and finance perspectives. Paris, 2015.

48 Moseley JB, O'Malley K, Petersen NJ, et al. A controlled trial of arthroscopic surgery for osteoarthritis of the knee. N Engl J Med 2002;347:81-8.

49 Mills EJ, loannidis JPA, Thorlund $\mathrm{K}$, et al. How to use an article reporting a multiple treatment comparison meta-analysis. JAMA 2012:308:1246.

50 Trinquart L, Attiche N, Bafeta A, et al. Uncertainty in treatment rankings: reanalysis of network meta-analyses of randomized trials. Ann Intern Med 2016;164:666.

51 Zhang J, Yuan Y, Chu H. The Impact of Excluding Trials from Network Meta-Analyses An Empirical Study. PLoS One 2016:11:e0165889.

52 Dias S, Caldwell DM. Network meta-analysis explained. Arch Dis Child Fetal Neonatal Ed 2019;104:F8-12. 